\title{
Polyethylene Glycol Wrapped Alginate/Graphene Hollow Microfibers as Flexible Supercapacitors
}

\author{
Vahid Nasirian ${ }^{1}$, Amir Ehsan Niaraki-Asli ${ }^{1}$, Saurabh S. Aykar ${ }^{1}$, Mehrnoosh Taghavimehr ${ }^{1}$, Reza \\ Montazami $^{1}$, and Nicole N. Hashemi ${ }^{1,2, a}$
}

1) Department of Mechanical Engineering, Iowa State University, Ames, IA, 50011 USA

2) Department of Mechanical Engineering, Stanford University, Stanford, CA, 94305 USA

a) Author to whom correspondence should be addressed; electronic mail: niolenh@ stanford.edu

\begin{abstract}
Carbon-modified fibrous structures with high biocompatibility have attracted much attention as supercapacitors due to their low cost, sustainability, abundance, and excellent electrochemical performance. However, some of these carbon-based materials suffer from low specific capacitance and electrochemical performance, which have been significant challenges in developing biocompatible electronic devices. In this regard, several studies have been reported on the development of 3D carbon-based micro architectures that provided high conductivity, energy storage potential, and 3D porosity frameworks. This study reports manufacturing of microfluidic Alginate hollow microfiber modified by water-soluble modified Graphene (BSA-Graphene). These architectures successfully exhibited conductivity enhancement conductivity of about 20 times more compared to Alginate hollow microfibers, and without any significant change in the inner-dimension values of hollow region $(220.0 \pm 10.0 \mu \mathrm{m})$ in comparison with pure alginate hollow microfibers. In the presence of Graphene, more obtained specific surface permeability and active ion adsorption sites could successfully provide as shorter pathways. These obtained continuous ion transport networks resulted in improved electrochemical performance. These desired electrochemical properties of the microfibers make Alginate/Graphene hollow fibers an excellent choice for further use in the development of lightweight flexible supercapacitors with scalable potential to be used in intelligent health electronic gadgets.
\end{abstract}


Keywords: Alginate/Graphene; hollow microfibers; Conductivity; Microfluidics; Supercapacitor.

\section{INTRODUCTION}

In the development of supercapacitors, one substantial key is the design of active nano and macro structures with high electrochemical performance. In this context, polymer fibers with small volume, lightweight, and high power density have attracted considerable attention in various electronic fields such as static charge dissipation, fiber-based portable, and wearable electronics storage [1-7], sensors [8-13], actuators [14], and electromagnetic interference shielding [15]. Various studies introducing metal filaments [16-20], intrinsically conductive polymers [21-23], carbon black [24], Graphite and Graphene [25], carbon nanotubes, and carbon nanofiber [26-29] as conductive modifiers [30, 31]. For improvement of the conductivity and charge storage potential of these fibers.

Various studies have been published on the successful modification of polymer fibers by Graphene to create conductive carbon nanoparticles/polymer composites with high biocompatibility and specific conductivity in many aspects of biomedical applications [32]. In fact, Graphene with a maximum number of covalentlybonded carbon atoms per mass unit could induce exceptional electrochemical behavior and more superficial porosity with shorter transport distances to these host polymer systems. Hence, ion accessibility of such constructions could be significantly enhanced. In this respect, a few fabrication techniques for the preparation of these conductive composite fibers such as microfluidics [13, 33-37], electrospinning [38, 39], wet-spinning [8, 12, 40, 41], melt-spinning [42-44], dry-film scrolling, [41] centrifugal spin [45], tube membrane ultrafiltration [46], and electrospinning [47], have been widely reported. Compared to other techniques, microfluidic is known as a critical pathway with high promising potentials for continuous fabrication of fibers at a large scale due to its high facility, and cost-effectiveness [48-53]. Hence, the microfluidic technique has played impressive roles in the development of single or multi-compartmental scaffolds in tissue engineering during recent years [54-57]. 
Moreover, conductive hollow microfibers with a significant surface to the volume value, have been developed by the microfluidic method, which were considered as high-performance supercapacitors for over applications ranging from energy to biomedical engineering [35, 58]. Since the electrochemical behavior of such fiber supercapacitors demands further investigation, a new design five-channel microfluidic device was employed in this report, to study on the continuous fabrication of conductive Alginate hollow microfibers through a mild gelation procedure and in the presence of $\mathrm{Ca}^{2+}$. As known, Alginate as the most popular natural biofunctional hydrogel has been widely used to fabricate hollow fibers with acceptable mechanical properties, and homogenous structure [59-63]. As far as we know, there are no previous reports about the employment of water-soluble bovine serum albumin (BSA)-Graphene as an electrically conductive modifier for Alginate hollow microfiber produced by five-channel microfluidic chip. In this respect, the manufacture Graphene percentage and flow rate ratio (FRR) between the core and sheath fluids on the morphology and the electrical conductivity properties of prepared hollow microfibers

were evaluated in detail. The obtained desired results could successfully confirm the potentials of microfluidic Alginate/Graphene hollow microfibers as a completely biocompatible structure, for achieving extraordinary capacitance and conductivity values in chip implants and intelligent electronic health gadgets.

\section{EXPERIMENTAL}

\section{A. Material}

$3.5 \%$ of alginate sodium solution (Product Number A18565) was prepared from Alfa Aesar, Ward Hill, MA. Poly (ethylene glycol) (PEG) $(\mathrm{Mn}=400$, Aldrich Chemistry, St. Louis, MO) contained 0.04\% $\mathrm{CaCl}_{2} \cdot 2 \mathrm{H}_{2} \mathrm{O}$ (Fisher Chemical, Waltham, MA) was used as the sheath solution. As a bath collector, an aqueous solution of $15 \% \mathrm{CaCl}_{2}$ was chosen. For the preparation of soluble Graphene, Graphite powder (Synthetic graphite powder $<20 \mu \mathrm{m}$,) and Bovine Serum Albumin BSA (A7906) were purchased from Aldrich Chemistry, St. Louis, MO. The employed AlTiN-coated end mill cutters and drill bits were purchased from Harvey tools and Grainger. Ultra-pure-water (18.2 M $\Omega . c m)$ was obtained from a Thermo 
Fisher Scientific water system (Waltham, MA). All chemicals used were of AR grades and used without further purification.

\section{B. Instrumental}

The electrical behaviors of the hollow microfibers were determined by Electrochemical Impedance Spectroscopy (EIS), Cyclic Voltammetry (CV), and Galvanostatic Charge/Discharge (GCD) and using a Potentiostat system (Versa STAT 4, Princeton Applied Research, Princeton, USA). A JCM-6000 NeoScope Benchtop scanning electron microscope (SEM) (Nisshin EM. CO., LTD, Tokyo, Japan) was used to study the manufactured hollow microfibers morphology. Differential Scanning Calorimetry Analysis (DSC) of the samples was carried out using the Mettler Toledo DSC instrument (Mettler Toledo 823 DSC, Switzerland), under a heating rate of $10{ }^{\circ} \mathrm{C} / \mathrm{min}$, and air atmosphere. The surface functional groups on the prepared samples were verified by a Fourier Transform Infrared Spectrometer (FTIR, PerkinElmer, Frontier, USA) over a range of $3500-750 \mathrm{~cm}^{-1}$. Three Lucca Technologies GenieTouch ${ }^{\mathrm{TM}}$ dual-channel syringe pumps were used to drive two $20 \mathrm{~mL}$ syringes.

\section{Preparation of BSA-Graphene Samples through Ball milling process}

The BSA-Graphene Samples were synthesized based on our previous method reported [64]. These FewLayer Graphene was fabricated through the Graphite crystallites' exfoliation procedure. For this aim, an aqueous mixture solution of Graphite $\left(20.00 \mathrm{mg} \mathrm{mL}^{-1}\right)$, and BSA $\left(2.00 \mathrm{mg} \mathrm{mL}^{-1}\right)$ was prepared in plastic containers where was contained a few steel balls with the diameter of $11 / 32^{\prime \prime}$ and $1 / 2^{\prime \prime}$ to apply for shear tensions at $300 \mathrm{rpm}$ rotational speed. The overall ratio of the balls was kept constant at $500 \pm 10 \mathrm{~m}^{2} / \mathrm{m}^{3}$ concerning the solution volume. The exfoliation process was followed by $90 \mathrm{~h}$ shaking after sealing and placing of the plastic containers inside metal containers.

\section{Fabrication of Microfluidic Devices}


According to our previous report [65], this five-channel microfluidic device used in this study to fabricate Alginate hollow microfibers, was manufactured from $6.0 \mathrm{~mm}$ thickness Poly (Methyl Methacrylate) (PMMA, Grainger, IL, US). A Computerized Numerical Control (CNC) mini-mill (Minitech Machinery Corporation, Norcross, GA) was used to mill the core channels and the two independent chevron chevrons with a dimension of $1.00 \mathrm{~mm} \times 0.75 \mathrm{~mm}$ (width $\times$ height) and $0.375 \mathrm{~mm} \times 0.25 \mathrm{~mm}($ width $\times$ height , respectively. Two faces of the PMMA chip were milled separately and then bonded together to obtain a microfluidic device.

\section{E. Fabrication of Alginate/Graphene hollow microfibers}

FIG. 1 shows the diagram of the microfluidic pathway developed in the current study. As can be seen, the syringe containing the core solution was connected to the center channel entrance as well as the sample and sheath solutions were respectively pumped into the middle and last twin outer channels of a five-channel microfluidic device by individual syringes. These solutions were flowed at a constant fluid flow rate of 200:450:230 $\mu \mathrm{L} / \mathrm{min}$ (sheath: core: sample) through their respective inlets and using the syringe pumps. Solid hollow microfibers could be formed from Alginate solution during a cross-linkage procedure in the presence of $\mathrm{Ca}^{2+}$. The core and sheath solutions remained in the water bath after solving in the $\mathrm{CaCl}_{2}$ solution. The produced hollow microfibers were collected using a set of tweezers and were safely mounted onto paper frames. These hollow microfibers were dried overnight at room temperature.

\section{Results and Discussion}

To develop an innovative method for the fabrication of conductive Alginate hollow microfibers, we fabricated a newly design five-channel microfluidic device equipped with two chevron regions to continuously manufacture these hollow microfibers, as shown in FIG. 1. As seen, its design contained a microchannel with three inlet channels converging onto the core channel owning two sets of four chevronshaped grooves extending vertically and twin three inlet channels located on either side. According to this design, Alginate composite hollow microfibers could be fabricated based on the rapid exchange and 
diffusion phenomena inside the microfluidic channel with a large specific surface area, and under a laminar flow regime. At the next step, the sheath fluid would join to these fluids to separate the polymer fluid from the walls, and wrapping these hollowfibers by PEG.

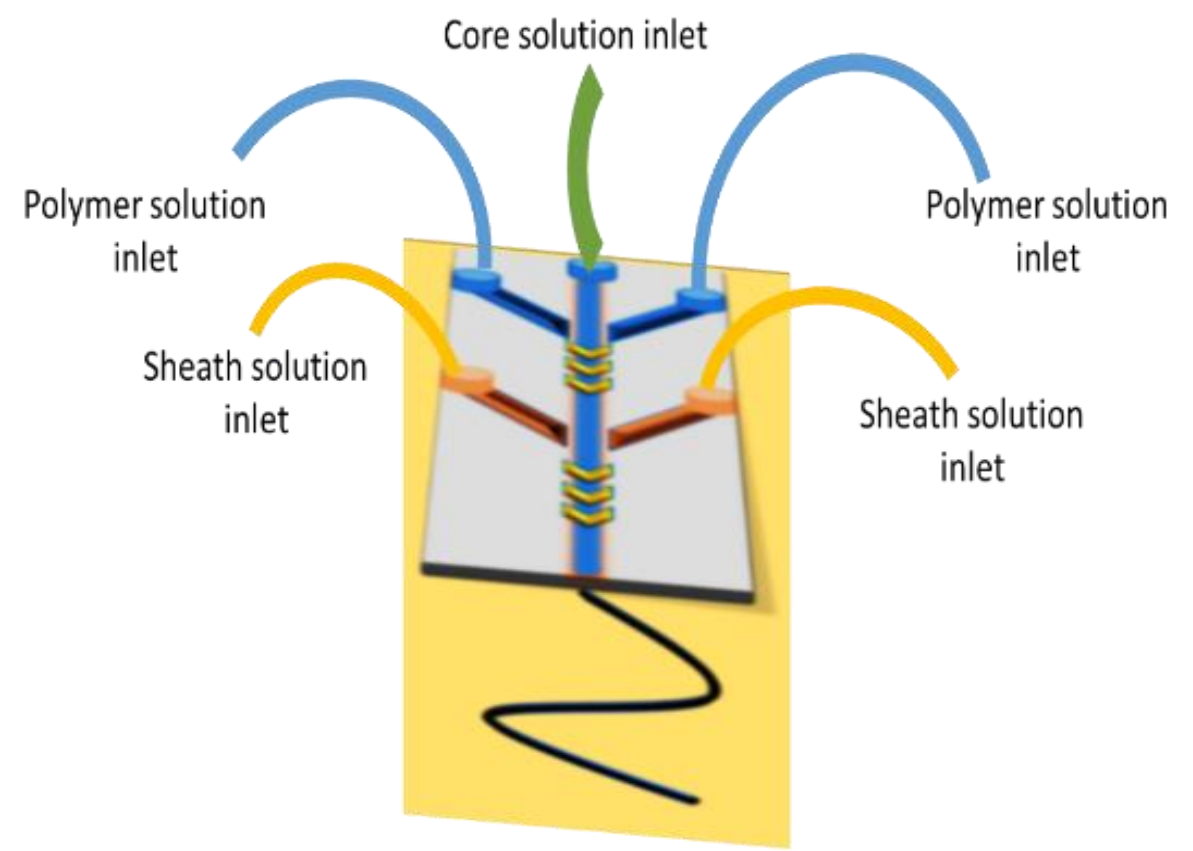

FIG. 1. Schematic of microfluidic procedure for Alginate/graphene hollow microfibers fabrication by five-channel microfluidic device.

Thus, the sizes of hollow microfluidic fibers could be completely influenced by the flow rate ratio (FRR) between employed solutions, which caused to provide a hydrodynamic focusing procedure along the microchannel sidewalls. Hence, detailed hollow dimensions of prepared microfibers at different FRR was investigated as the most crucial efficient parameter (FIG. 2.) 


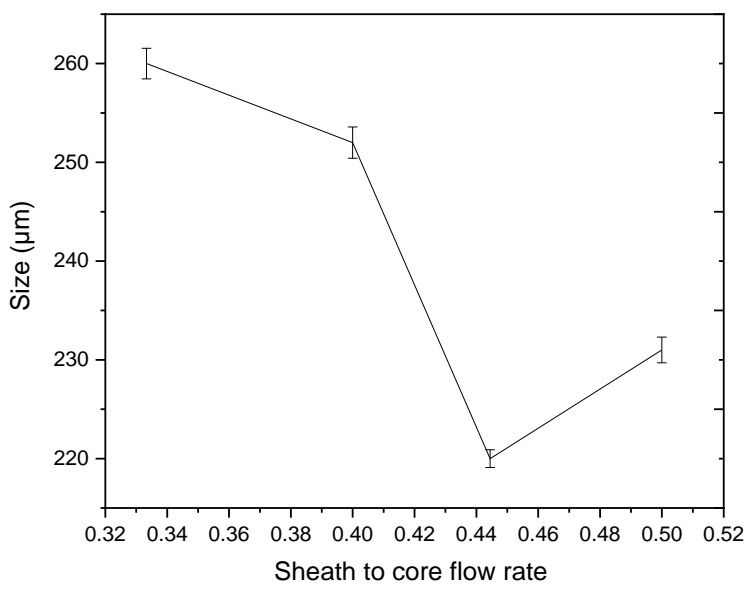

FIG. 2. Study of dependency of hollow size dimensions of Alginate microfibers to sheath-to-core flow rate ratios. These results were obtained under $0.50,0.44,0.40$ and 0.33 FRR and data are presented as mean of five measurements $( \pm \mathrm{SD})$. The polymer flow was remained constant at $230.0 \mu \mathrm{L} / \mathrm{min}$.

As seen, the hollow size decreased from $260.0 \mu \mathrm{m}$ to $220.0 \mu \mathrm{m}$ by increasing the FRR from 0.33 to 0.45 due to the enhancement of hydrodynamic focusing by FRR increasing. At lower flow rate ratio, the applied hydrodynamic focusing on polymer fluid has been decreased. As a result, the growing of hollow fibers could be occupied in the lateral direction. At FRR of 200.0: 450.0: $230.0 \mu \mathrm{L} / \mathrm{min}$ (sheath:core:sample) point, the maximum hydrodynamic focusing could be prepared on Alginate fluid for construction of microfluidic Alginate hollow microfibers, which was chosen as the optimum FRR point for subsequent steps. After the addition of BSA-Graphene to Alginate solution, the viscosity of the obtained polymer fluid increased resulting in a weakened hydrodynamic focusing from the core flow. Therefore, the FRR for Alginate/Graphene had been insignificantly shifted to smaller values to compensate for this weakness. The prepared hollow microfibers morphology was analyzed by SEM to determine the sizes of these hollow microfibers samples (FIG. 3). As shown in FIG. 3, the average inner-dimension value was obtained as about $220.0 \pm 10.0 \mu \mathrm{m}$ for free Alginate and Alginate/graphene hollow microfibers, and no noticeable changes were observed in sizes of Alginate hollow microfibers after Graphene introducing to polymer solutions. 

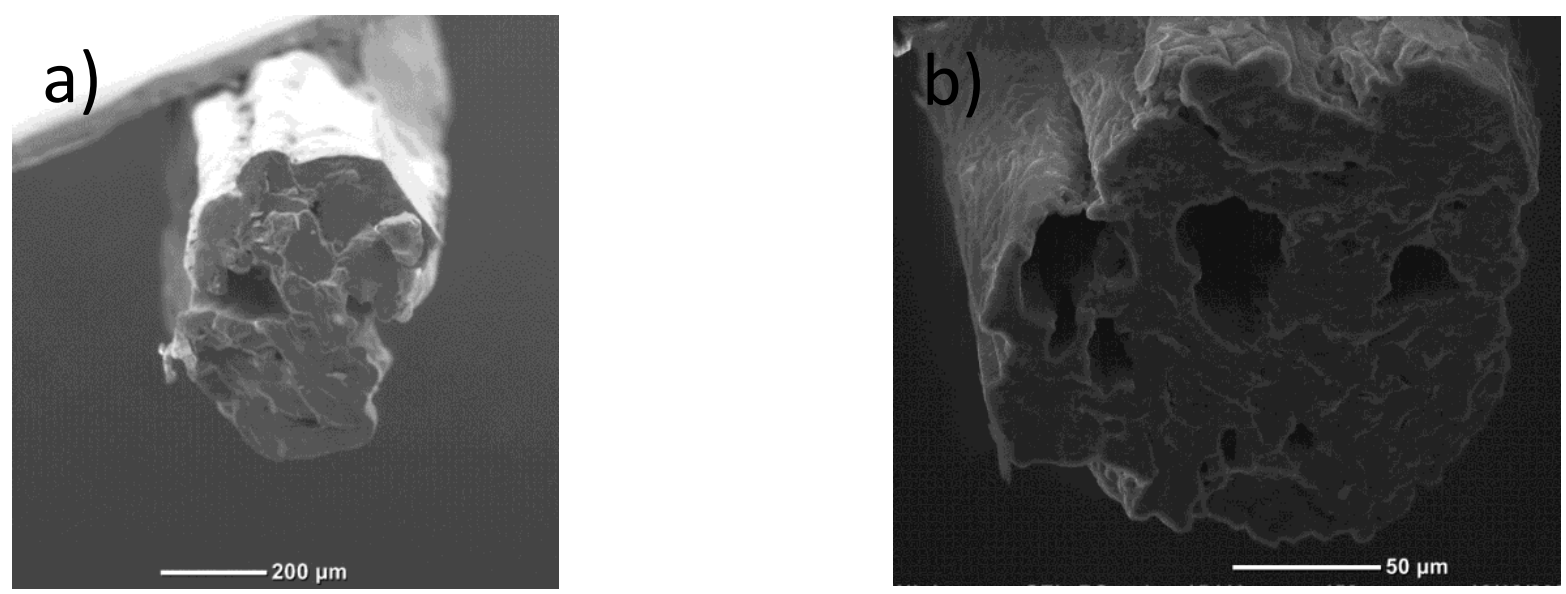

FIG. 3. The morphology of hollow microfibers (a) Alginate and (b) modified Alginate/graphene manufactured under optimum FRR.

In addition, the incorporation graphene into Alginate did not significantly change the morphology of the fibers. Due to the different degrees of contraction between Alginates and Alginate/Graphene particles during gelation procedure, both samples showed a porous manner on their inner and outer surface. The related chemical characterizations of this synthesized BSA-Graphene were described in our previous report [64]. To study the melting behavior of the Alginate and Alginate/Graphene hollow microfibers wrapped by PEG, related thermal analysis was determined by DSC. The thermal results obtained under a heating flow rate of $10{ }^{\circ} \mathrm{C} / \mathrm{min}$, are presented in FIG. 4. As seen, these DSC thermograms exhibit one endothermic peak at $\sim 85{ }^{\circ} \mathrm{C}$ related to the evaporation of water molecules and the oxidative degradation of Alginate polymers and their calcium-carboxylate bonds as an exothermic peak at $\sim 251^{\circ} \mathrm{C}$ [66].

The Elasticity Characterization of Alginate Fibers was studied under different FRRs, and $\mathrm{CaCl}_{2}$ concentrations were reported in our previously published articles. These results expressed that the obtained Young's modulus values (MPa) for these fibers could be enhanced up to about $75 \mathrm{MPa}$ under optimum conditions. In addition, we showed that the Tensile Stress amount obtained for microfluidic Alginate fibers created under various FRR, could be enhanced 10 times more ( 15 $\mathrm{MPa})$ by increasing the $\mathrm{CaCl}_{2}$ concentration from $0 \%$ to $5 \%$ [67].

In addition, these DSC thermograms displayed sharp melting endothermic peaks at $\sim 65^{\circ} \mathrm{C}$ related to PEG as an employed capping layer. For the Alginate/Graphene hollow microfiber, a distinct endothermic was 
observed at $\sim 350{ }^{\circ} \mathrm{C}$ that is related to oxygen elimination reactions of carbon atoms fundable in employed Graphene structure, which could successfully confirm the presence of Graphene inside the preparation of hollow microfibers without any significant impact on the crystal structure of Alginate hollow microfibers.

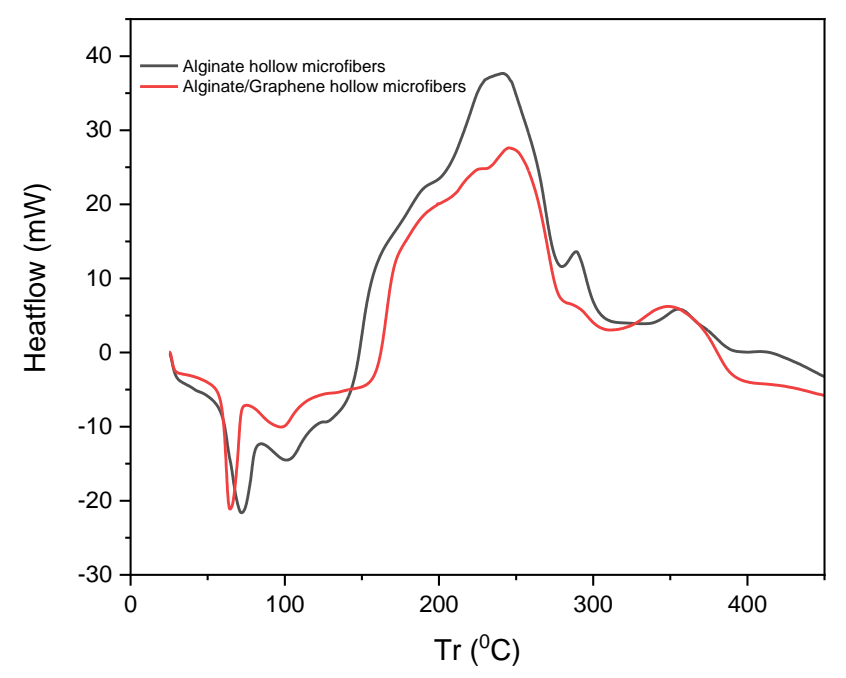

FIG. 4. DSC thermograms of Alginate and Alginate/Graphene hollow microfibers wrapped by PEG.

The surface factor groups that appeared during the formation of the Alginate hollow microfiber were characterized by FT-IR spectroscopy method. FIG. 5 shows a comparison between the FTIR spectra related to Alginate hollow microfibers before and after the addition of Graphene.

It is evident that the characteristic broad absorption band around $3350 \mathrm{~cm}^{-1}$ corresponding to the $\mathrm{O}-\mathrm{H}$ stretching, the $\mathrm{C}-\mathrm{H}$ stretching bond groups of $\mathrm{O}-\mathrm{C}-\mathrm{H}$ group functions are apparent in the range from 2800 to $2900 \mathrm{~cm}^{-1}$, and the $-\mathrm{C}-\mathrm{O}-$ stretching are apparent from 1000 to $1200 \mathrm{~cm}^{-1}$. These results are providing clear evidence for covering of these hollow microstructures by PEG. Observed bands in $1627 \mathrm{~cm}^{-1}$ and $1465 \mathrm{~cm}^{-1}$ were attributed to asymmetric and symmetric stretching vibrations of the linkage between $\mathrm{Ca}$ and carboxylate functional group of Alginate, respectively. 


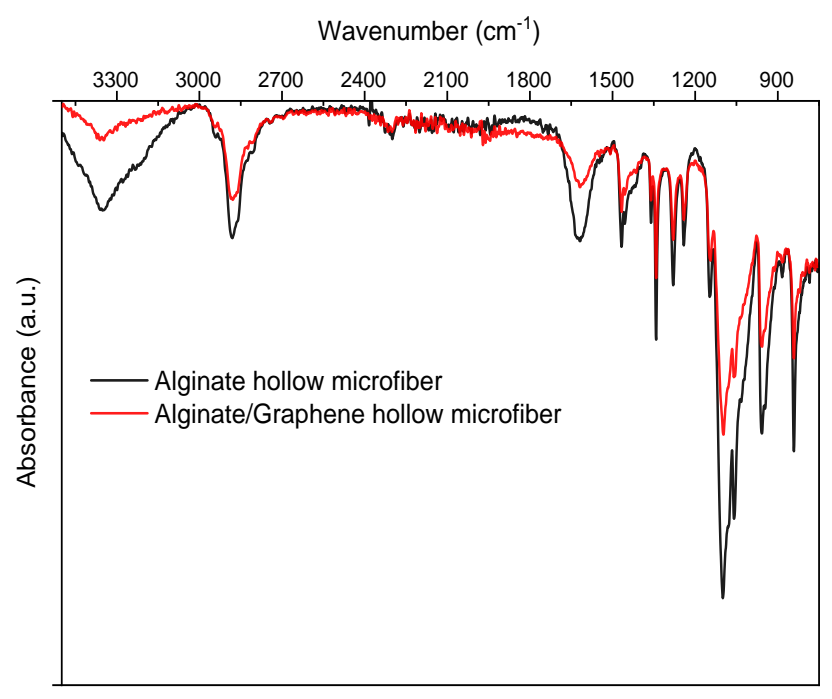

FIG. 5. FT-IR spectra of developed PEG wrapped Alginate and Alginate/Graphene hollow microfiber.

In addition, the electrical conductivity of the prepared microfluidic Alginate hollow microfibers was investigated through the $\mathrm{CV}$ method and at the potential range -1.0 to $+1.0 \mathrm{~V}$ as a dependent factor to Graphene amount (FIG. 6 (a)).

In this order, two fibers (length $\approx 5 \mathrm{~cm}$ ) were arranged in parallel on a Polyethylene terephthalate (PET) substrate with about $5 \mathrm{~mm}$ a distancing. The endpoints of these hollow fibers were adhered to the PET substrate by silver paste. These Alginate/Graphene hollow microfiber were carefully coated with a thin layer of $\mathrm{H}_{3} \mathrm{PO}_{4} / \mathrm{PVA}$ gel electrolyte which was prepared as follows:

1.0 gr $\mathrm{H}_{3} \mathrm{PO}_{4}$ and $1.0 \mathrm{gr}$ PVA were mixed with $10.0 \mathrm{~mL}$ ultrapure water and vigorously stirred at $80{ }^{\circ} \mathrm{C}$ for 3 hours to obtain a homogeneous and transparent gel. As a result, versatile solid-state fiber-shaped electrode could be prepared by filling $\mathrm{H}_{3} \mathrm{PO}_{4} / \mathrm{PVA}$ into the middle overlapped and then dried at room temperature.

FIG. 6 (a) shows the representative CV results for the manufactured Alginate hollow microfibers contained various percentage of Graphene in the potential range from -1.0 to $+1.0 \mathrm{~V}$ (vs. $\mathrm{Ag} / \mathrm{AgCl}$ ) at a scan rate of $10.0 \mathrm{mV} . \mathrm{s}^{-1}$ and $21^{\circ} \mathrm{C}$. 

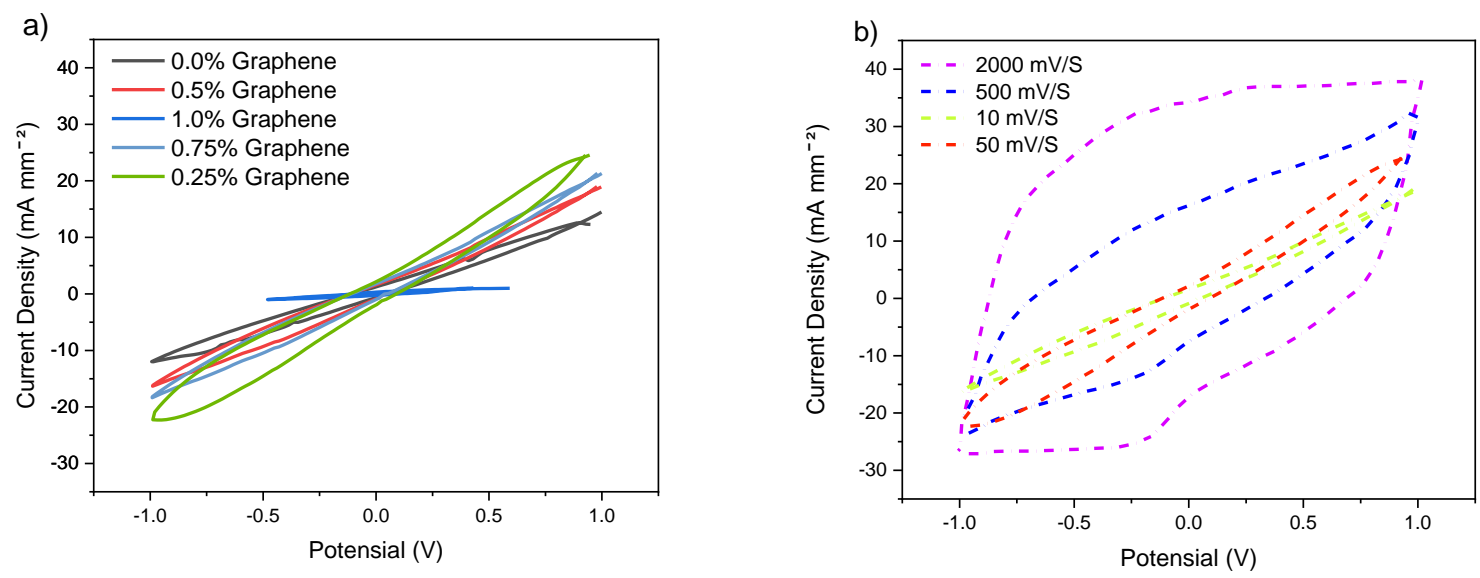

FIG. 6. (a) Characterization of the Alginate hollow microfibers contained different amount of Graphene through $\mathrm{CV}$ method in the potential range from -1.0 to $+1.0 \mathrm{~V}$ (vs. $\mathrm{Ag} / \mathrm{AgCl}$ ) and at a scan rate of 10.0 $\mathrm{mV} \mathrm{s}^{-1}$. (b) Electrical behavior of conductive Alginate/Graphene hollow microfibers at various scan rates $\left(10.0-2000.0 \mathrm{mV} \mathrm{s}^{-1}\right)$.

As seen, these peak current related to Alginate/Graphene hollow microfibers CV curves improved by almost a range factor $10-25$, and the voltage window increased, from $0.5 \mathrm{~V}$ to $1.0 \mathrm{~V}$ at a $10.0 \mathrm{mV} \mathrm{s}^{-1} \mathrm{scan}$ rate, which could successfully confirm the induction of electrical conductivity to Alginate hollow microfibers as a result of employing of Graphene.

In comparison to pure Alginate samples, the broader peak area and a nearly rectangular behavior with high stability was exhibited by Alginate/Graphene hollow microfibers CV results could be considered as effects of electric double-layer series. Moreover, an acceptable energy-storage ability, and effective ion transporting in the prepared capacitance spots gained by Graphene introduced into Alginate hollow microfibers.

Unexpectedly, the increasing the graphene amounts more than $0.25 \%$ did not provide any more conductivity enhancement for the development Alginate/Graphene hollow microfibers that could be due to the loss porosity manner of these structures by adding the Graphene percentage.

For further confirmation of the charge transmission and ion transport enhancement obtained from the developed Alginate structures, the percentage of $0.25 \%$ Graphene as an optimum amount of a conductive 
modifier, the $\mathrm{CV}$ characterization was continued under various the scanning rate from 10.0 to $2000.0 \mathrm{mV}$ $\mathrm{s}^{-1}$. As seen in FIG. 6 (b), the CV plots became more eye-shaped by increasing the scan rate up to 2000.0 $\mathrm{mV} \mathrm{s}^{-1}$ due to the occurred limitations for ions mobility in the $\mathrm{PVA} / \mathrm{H}_{2} \mathrm{SO}_{4}$ gel electrolyte during the fast voltage variation at a high scan rate. In addition, these obtained results clearly showed the stability related of the electric double-layer capacitance related to hollow microfibers over the whole voltage range.

Furthermore, the dielectric, ion-transport behaviors and ion kinetics manners of the prepared microfluidic Alginate hollow microfibers before and after the addition of Graphene to this structure were more investigated by EIS technique. As shown in FIG. 7 (a), the obtained Nyquist plots were comprised of semicircular curved reigns that obtained in two regions: the high and medium frequency region referred to the total ohmic resistance or the charge-transfer reaction resistance. Moreover, the second trailed straight lines curves that appeared in the low frequency region could reflect the diffusion process inside these structures.

These straight lines obtained in the low frequency range for Alginate/Graphene hollow microfibers samples show higher rates (slopes) compared to Alginate samples due to higher availability of electro active sites inside Alginate/Graphene hollow microfibers.

As a result of the more prepared porosity, and ions transporting pathways for the Alginate/Graphene hollow microfibers, greater ion diffusion manner to Alginate hollow microfiber samples obtained by the added Graphene as conductive modifier [68]. In addition, the Alginate/Graphene hollow microfibers samples successfully exhibited less $\left|Z^{\prime \prime}\right|$ in the high frequency range with their enhanced conductivity compared to Alginate hollow microfiber. However, this upward trend had not been achieved for Alginate/Graphene hollow microfiber by increasing the Graphene amount from 0.5 to $1.0 \%$ because of losing the obtained porosity manner at these percentages.

The specific capacitance behavior of prepared hollow microfibers was investigated as the most important features to confirm their supercapacitor manner by galvanostatic charge/discharge technique under current densities (j) of $0.5 \mathrm{~A} / \mathrm{g}$. The results obtained of this study are shown in FIG. 7 (b). 
a)

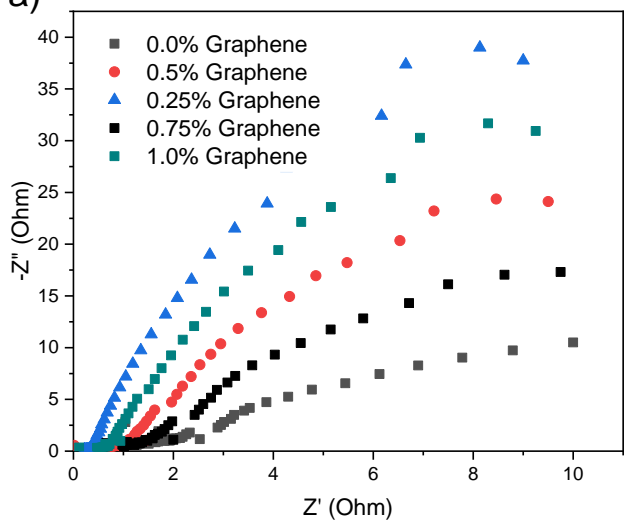

b)

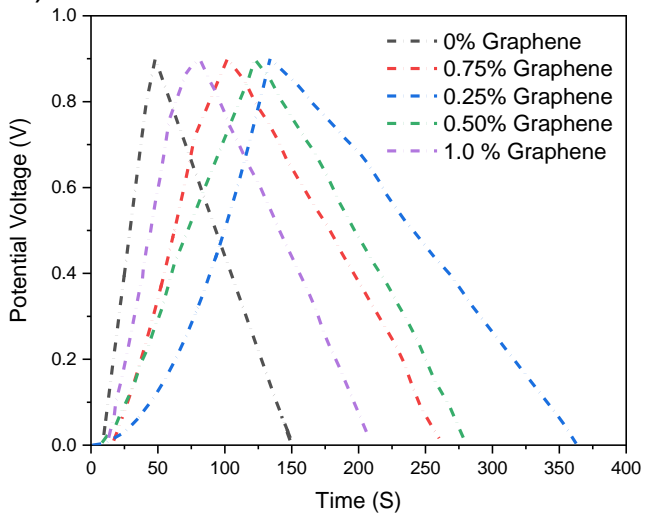

FIG. 7. (a) Nyquist plots of the prepared hollow fibers in the frequency range from $100 \mathrm{kHz}$ to $0.01 \mathrm{~Hz}$. (b) Galvanostatic charge/discharge curves of the Alginate and Alginate/graphene hollow microfibers series at the current density of $0.5 \mathrm{~A} \mathrm{~g}^{-1}$.

As seen, the Alginate hollow microfibers samples exhibited an enhancement in charge/discharge manner at $0.5 \mathrm{~A} / \mathrm{g}$ with symmetry and triangular behavior.

By adding the Graphene percentage from 0-05\%, Alginate/Graphene hollow microfiber could express more benefit in charge/discharge procedures with large peak current and a long discharging time, and no obvious ohmic loss (IR drop) at the low current densities in compared to Alginate Hollow fibers.

This is because of the provided sufficient electrical double layer capacitance with larger effective surface area as ion-accessible path channels for the rapid motion of electrons inside this mesoporous structure.

However, Alginate/Graphene hollow microfibers demonstrated more IR at $j=0.5 \mathrm{~A} / \mathrm{g}$ by addition of Graphene percentage from $0.25 \%$ to $1.0 \%$, which resulted in the loss of porosity manner of generated Alginate/Graphene hollow microfibers.

As seen, the discharging time obtained was longer than the charge time for samples contained Graphene. The short circuits founded in the developed microfiber electrode associated with the discharging procedure of these charged capacitors. However, their related resistances could slightly increase the equivalent resistance of the whole system, which eventually led to a slightly lengthened time in discharging time according to the direct relationship between the discharging time and the equivalent resistance as following: 
$V_{C}=V_{S}\left(1-e^{\left(-\frac{t}{R C}\right)}\right)$

Eq. 1

Where $V c$ is the voltage across the capacitor, $V s$ is the supply voltage, $\mathrm{t}$ is the elapsed time since the application of the supply voltage, $R$ is the Internal resistance and $C$ is the capacitor capacity.

\section{CONCLUSION}

In this study, electrically conductive Alginate/graphene composite hollow microfibers were fabricated for the first time using a microfluidic approach. Morphology, conductivity, and other composite fibers' features were studied under various flow rate ratios and graphene concentrations. According to obtained results, 200.0: 450.0: $230.0 \mu \mathrm{L} / \mathrm{min}$ (sheath:core: sample) and 0.25\% were chosen as optimum points for FRR and graphene concentration, respectively. In conclusion, microfluidic technology could successfully confirm itself as a functional approach for fabricating electrically-conductive Alginate/graphene hollow microfibers with about ten times more conductivity Alginate hollow microfibers and controllable size and shape compared to other fiber preparation techniques. Moreover, this work may open up more opportunities for such Alginate/Graphene hollow microfibers having significant potentials to be employed in various areas such as energy, biomedical applications, and single-cell analysis. Future work could include the study of these Alginate / Graphene hollow microfibers potentials in the development of hollow microvessel fibers according to current requirements in specific physiological hollow microvessel and tissue engineering.

\section{Conflict of Interest}

There are no conflicts to declare.

\section{Data Availability Statement}

The data that support the findings of this study are available from the corresponding author upon reasonable request. 


\section{ACKNOWLEDGMENTS}

This work was partially supported by the Office of Naval Research Grant N000141712620 and National

Science Foundation Award 2014346.

\section{REFERENCES}

[1] Pu, X., Li, L., Liu, M., Jiang, C., Du, C., Zhao, Z., Hu, W., and Wang, Z. L., "Wearable self-charging power textile based on flexible yarn supercapacitors and fabric nanogenerators," Adv. Mater. 28, 98-105 (2016).

[2] Kou, L., Huang, T., Zheng, B., Han, Y., Zhao, X., Gopalsamy, K., Sun, H., and Gao, C., "Coaxial wet-spun yarn supercapacitors for high-energy density and safe wearable electronics," Nat. Commun. 5, 1-10 (2014).

[3] Liu, L., Yu, Y., Yan, C., Li, K., and Zheng, Z., "Wearable energy-dense and power-dense supercapacitor yarns enabled by scalable graphene-metallic textile composite electrodes," Nat. Commun. 6, 1-9 (2015). [4] Yu, D., Goh, K., Wang, H., Wei, L., Jiang, W., Zhang, Q., Dai, L., and Chen, Y., "Scalable synthesis of hierarchically structured carbon nanotube-graphene fibres for capacitive energy storage," Nat.

Nanotechnol. 9, 555 (2014).

[5] Zhang, Z., Wang, L., Li, Y., Wang, Y., Zhang, J., Guan, G., Pan, Z., Zheng, G., and Peng, H., "Nitrogendoped core-sheath carbon nanotube array for highly stretchable supercapacitor," Adv. Energy Mater. 7, 1601814 (2017).

[6] Zeng, W., Shu, L., Li, Q., Chen, S., Wang, F., and Tao, X. M., "Fiber-based wearable electronics: a review of materials, fabrication, devices, and applications," Adv. Mater. 26, 5310-5336 (2014).

[7] Bao, L., and Li, X., "Towards textile energy storage from cotton T-shirts," Adv. Mater. 24, 3246-3252 (2012).

[8] Seyedin, S., Razal, J. M., Innis, P. C., Jeiranikhameneh, A., Beirne, S., and Wallace, G. G., "Knitted strain sensor textiles of highly conductive all-polymeric fibers," ACS Appl. Mater. Interfaces 7, 2115021158 (2015).

[9] Bera, T. K., Mohamadou, Y., Lee, K., Wi, H., Oh, T. I., Woo, E. J., Soleimani, M., and Seo, J. K., "Electrical impedance spectroscopy for electro-mechanical characterization of conductive fabrics," Sensors 14, 9738-9754 (2014).

[10] Cherenack, K., Zysset, C., Kinkeldei, T., Münzenrieder, N., and Tröster, G., "Woven electronic fibers with sensing and display functions for smart textiles," Adv. Mater. 22, 5178-5182 (2010).

[11] Li, C., Thostenson, E. T., and Chou, T.-W., "Sensors and actuators based on carbon nanotubes and their composites: a review," Compos. Sci. Technol. 68, 1227-1249 (2008).

[12] Cochrane, C., Lewandowski, M., and Koncar, V., "A flexible strain sensor based on a conductive polymer composite for in situ measurement of parachute canopy deformation," Sensors 10, 8291-8303 (2010).

[13] Hashemi, N., Lackore, J. M., Sharifi, F., Goodrich, P. J., Winchell, M. L., and Hashemi, N., "A paperbased microbial fuel cell operating under continuous flow condition," TECHNOLOGY 4, 98-103 (2016).

[14] Qi, B., Lu, W., and Mattes, B. R., "Strain and energy efficiency of polyaniline fiber electrochemical actuators in aqueous electrolytes," J. Phys. Chem. B 108, 6222-6227 (2004).

[15] Shinagawa, S., Kumagai, Y., and Urabe, K., "Conductive papers containing metallized polyester fibers for electromagnetic interference shielding," J. Porous Mater. 6, 185-190 (1999). 
[16] Tao, J., Liu, N., Ma, W., Ding, L., Li, L., Su, J., and Gao, Y., "Solid-state high performance flexible supercapacitors based on polypyrrole-MnO 2-carbon fiber hybrid structure," Sci. Rep. 3, 2286 (2013).

[17] Devaraj, S., and Munichandraiah, N., "Effect of crystallographic structure of MnO2 on its electrochemical capacitance properties," J. Phys. Chem. C 112, 4406-4417 (2008).

[18] Li, D., Gong, Y., Wang, M., and Pan, C., "Preparation of sandwich-like NiCo $2 \mathrm{O}$ 4/rGO/NiO heterostructure on nickel foam for high-performance supercapacitor electrodes," Nanomicro Lett. 9, 16 (2017).

[19] Wu, P., Cheng, S., Yao, M., Yang, L., Zhu, Y., Liu, P., Xing, O., Zhou, J., Wang, M., and Luo, H., “A lowcost, self-standing NiCo2O4@ CNT/CNT multilayer electrode for flexible asymmetric solid-state supercapacitors," Adv. Funct. Mater. 27, 1702160 (2017).

[20] Zhu, Y., Zhao, Y., Zhang, X., Wang, L., Wang, X., Zhang, J., Han, P., and Qiao, J., "Metal

filaments/nano-filler filled hybrid polymer fibers with improved conductive performance," Mater. Lett. 173, 26-30 (2016).

[21] Tang, L., Du, D., Yang, F., Liang, Z., Ning, Y., Wang, H., and Zhang, G.-J., "Preparation of GrapheneModified Acupuncture Needle and Its Application in Detecting Neurotransmitters," Sci. Rep. 5, 11627 (2015).

[22] Spearman, B. S., Hodge, A. J., Porter, J. L., Hardy, J. G., Davis, Z. D., Xu, T., Zhang, X., Schmidt, C. E., Hamilton, M. C., and Lipke, E. A., "Conductive interpenetrating networks of polypyrrole and polycaprolactone encourage electrophysiological development of cardiac cells," Acta Biomater. 28, 109120 (2015).

[23] Acar, H., Çınar, S., Thunga, M., Kessler, M. R., Hashemi, N., and Montazami, R., "Study of physically transient insulating materials as a potential platform for transient electronics and bioelectronics," Adv. Funct. Mater. 24, 4135-4143 (2014).

[24] JIN, X. X., and XIE, C., "Non-continuous conductive behavior of CB/PET fiber," Chin. J. Mater. Res. 24, 311-314 (2010).

[25] Sayyar, S., Murray, E., Thompson, B. C., Gambhir, S., Officer, D. L., and Wallace, G. G., "Covalently linked biocompatible graphene/polycaprolactone composites for tissue engineering," Carbon 52, 296304 (2013).

[26] Jain, R., Chae, H. G., and Kumar, S., "Polyacrylonitrile/carbon nanofiber nanocomposite fibers," Compos. Sci. Technol. 88, 134-141 (2013).

[27] Sanchez-Garcia, M., Lagaron, J., and Hoa, S., "Effect of addition of carbon nanofibers and carbon nanotubes on properties of thermoplastic biopolymers," Compos. Sci. Technol. 70, 1095-1105 (2010).

[28] Jeong, J. S., Moon, J.-S., Jeon, S. Y., Park, J. H., Alegaonkar, P. S., and Yoo, J. B., "Mechanical properties of electrospun PVA/MWNTs composite nanofibers," Thin Solid Films 515, 5136-5141 (2007). [29] Chien, A.-T., Gulgunje, P. V., Chae, H. G., Joshi, A. S., Moon, J., Feng, B., Peterson, G., and Kumar, S., "Functional polymer-polymer/carbon nanotube bi-component fibers," Polymer 54, 6210-6217 (2013). [30] Yu, Y., Song, S., Bu, Z., Gu, X., Song, G., and Sun, L., "Influence of filler waviness and aspect ratio on the percolation threshold of carbon nanomaterials reinforced polymer nanocomposites," J. Mater. Sci. 48, 5727-5732 (2013).

[31] Yi, J. Y., and Choi, G. M., "Percolation behavior of conductor-insulator composites with varying aspect ratio of conductive fiber," J. Electroceram. 3, 361-369 (1999).

[32] McNamara, M. C., Niaraki-Asli, A. E., Guo, J., Okuzono, J., Montazami, R., and Hashemi, N. N., "Enhancing the Conductivity of Cell-Laden Alginate Microfibers With Aqueous Graphene for Neural Applications," Front. Mater. Sci. 7, 61 (2020).

[33] Mitrovski, S. M., Elliott, L. C., and Nuzzo, R. G., "Microfluidic devices for energy conversion: Planar integration and performance of a passive, fully immersed H2- 02 fuel cell," Langmuir 20, 6974-6976 (2004). 
[34] Sharifi, F., Ghobadian, S., Cavalcanti, F. R., and Hashemi, N., "based devices for energy applications," Renew. Sustain. Energy Rev. 52, 1453-1472 (2015).

[35] Sharifi, F., Sooriyarachchi, A. C., Altural, H., Montazami, R., Rylander, M. N., and Hashemi, N., "Fiber based approaches as medicine delivery systems," ACS Biomater. Sci. Eng. 2, 1411-1431 (2016).

[36] Goodrich, P. J., Sharifi, F., and Hashemi, N., "Rapid prototyping of microchannels with surface patterns for fabrication of polymer fibers," RSC Adv. 5, 71203-71209 (2015).

[37] Sharifi, F., Bai, Z., Montazami, R., and Hashemi, N., "Mechanical and physical properties of poly (vinyl alcohol) microfibers fabricated by a microfluidic approach," RSC Adv. 6, 55343-55353 (2016).

[38] Sundaray, B., Subramanian, V., Natarajan, T., and Krishnamurthy, K., "Electrical conductivity of a single electrospun fiber of poly (methyl methacrylate) and multiwalled carbon nanotube nanocomposite," Appl. Phys. Lett. 88, 143114 (2006).

[39] Ghasemi-Mobarakeh, L., Prabhakaran, M. P., Morshed, M., Nasr-Esfahani, M. H., Baharvand, H., Kiani, S., Al-Deyab, S. S., and Ramakrishna, S., "Application of conductive polymers, scaffolds and electrical stimulation for nerve tissue engineering," J. Tissue Eng. Regen. Med. 5, e17-e35 (2011). [40] Xue, P., Park, K., Tao, X., Chen, W., and Cheng, X., "Electrically conductive yarns based on PVA/carbon nanotubes," Compos. Struct. 78, 271-277 (2007).

[41] Cruz-Silva, R., Morelos-Gomez, A., Kim, H.-i., Jang, H.-k., Tristan, F., Vega-Diaz, S., Rajukumar, L. P., Elías, A. L., Perea-Lopez, N., and Suhr, J., "Super-stretchable graphene oxide macroscopic fibers with outstanding knotability fabricated by dry film scrolling," ACS nano 8, 5959-5967 (2014).

[42] Kim, B., Koncar, V., Devaux, E., Dufour, C., and Viallier, P., "Electrical and morphological properties of PP and PET conductive polymer fibers," Synth. Met. 146, 167-174 (2004).

[43] Lim, T. H., Lee, S. H., and Yeo, S. Y., "Highly conductive polymer/metal/carbon nanotube composite fiber prepared by the melt-spinning process," Text. Res. J. 87, 593-606 (2017).

[44] Fornes, T., Baur, J., Sabba, Y., and Thomas, E., "Morphology and properties of melt-spun polycarbonate fibers containing single-and multi-wall carbon nanotubes," Polymer 47, 1704-1714 (2006).

[45] Lu, Y., Fu, K., Zhang, S., Li, Y., Chen, C., Zhu, J., Yanilmaz, M., Dirican, M., and Zhang, X., “Centrifugal spinning: a novel approach to fabricate porous carbon fibers as binder-free electrodes for electric double-layer capacitors," J. Power Sources 273, 502-510 (2015).

[46] Yu, Y., Zhong, J., Sun, W., Kumar, R., and Koratkar, N., "Solid-State Hybrid Fibrous Supercapacitors Produced by Dead-End Tube Membrane Ultrafiltration," Adv. Funct. Mater. 27, 1606461 (2017).

[47] Simotwo, S. K., DelRe, C., and Kalra, V., "Supercapacitor electrodes based on high-purity electrospun polyaniline and polyaniline-carbon nanotube nanofibers," ACS Appl. Mater. Interfaces 8 , 21261-21269 (2016).

[48] Wu, G., Tan, P., Wu, X., Peng, L., Cheng, H., Wang, C. F., Chen, W., Yu, Z., and Chen, S., "HighPerformance Wearable Micro-Supercapacitors Based on Microfluidic-Directed Nitrogen-Doped Graphene Fiber Electrodes," Adv. Funct. Mater. 27, 1702493 (2017).

[49] Xu, T., Ding, X., Liang, Y., Zhao, Y., Chen, N., and Qu, L., "Direct spinning of fiber supercapacitor," Nanoscale 8, 12113-12117 (2016).

[50] Bai, Z., Reyes, J. M. M., Montazami, R., and Hashemi, N., "On-chip development of hydrogel microfibers from round to square/ribbon shape," J. Mater. Chem. A . 2, 4878-4884 (2014).

[51] Sharifi, F., Patel, B. B., McNamara, M. C., Meis, P. J., Roghair, M. N., Lu, M., Montazami, R., Sakaguchi, D. S., and Hashemi, N. N., "Photo-cross-linked poly (ethylene glycol) diacrylate hydrogels: spherical microparticles to bow tie-shaped microfibers," ACS Appl. Mater. Interfaces 11, 18797-18807 (2019).

[52] Caplin, J. D., Granados, N. G., James, M. R., Montazami, R., and Hashemi, N., "Microfluidic organ-ona-chip technology for advancement of drug development and toxicology," Adv. Healthc. Mater. 4, 14261450 (2015). 
[53] Pemathilaka, R. L., Caplin, J. D., Aykar, S. S., Montazami, R., and Hashemi, N. N., "Placenta-on-aChip: In Vitro Study of Caffeine Transport across Placental Barrier Using Liquid Chromatography Mass Spectrometry," GLOB. CHALL. 3, 1800112 (2019).

[54] Zuo, Y., He, X., Yang, Y., Wei, D., Sun, J., Zhong, M., Xie, R., Fan, H., and Zhang, X., "Microfluidicbased generation of functional microfibers for biomimetic complex tissue construction," Acta Biomater. 38, 153-162 (2016).

[55] Bettinger, C. J., "Synthesis and microfabrication of biomaterials for soft-tissue engineering," Pure Appl. Chem. 81, 2183-2201 (2009).

[56] Ma, J., Wang, Y., and Liu, J., "Biomaterials meet microfluidics: from synthesis technologies to biological applications," Micromachines 8, 255 (2017).

[57] Chung, B. G., Lee, K.-H., Khademhosseini, A., and Lee, S.-H., "Microfluidic fabrication of microengineered hydrogels and their application in tissue engineering," Lab. Chip 12, 45-59 (2012). [58] Sechi, D., Greer, B., Johnson, J., and Hashemi, N., "Three-dimensional paper-based microfluidic device for assays of protein and glucose in urine," Anal. Chem. 85, 10733-10737 (2013).

[59] Lee, K. H., Shin, S. J., Park, Y., and Lee, S. H., "Synthesis of cell-laden alginate hollow fibers using microfluidic chips and microvascularized tissue-engineering applications," Small 5, 1264-1268 (2009). [60] Nie, M., and Takeuchi, S., "Microfluidics based synthesis of coiled hydrogel microfibers with flexible shape and dimension control," Sensors Actuators B: Chem. 246, 358-362 (2017).

[61] Yoshida, K., and Onoe, H., "Functionalized core-shell hydrogel microsprings by anisotropic gelation with bevel-tip capillary," Sci. Rep. 7, 1-9 (2017).

[62] Yi, B., Shim, K. Y., Ha, S. K., Han, J., Hoang, H.-H., Choi, I., Park, S., and Sung, J. H., "Threedimensional in vitro gut model on a villi-shaped collagen scaffold," BioChip J. 11, 219-231 (2017).

[63] Lee, Y., and Park, J.-K., "Microfabricated cell culture system for the live cell observation of the multilayered proliferation of undifferentiated HT-29 cells," BioChip J. 11, 308-315 (2017).

[64] Guo, J., Niaraki Asli, A. E., Williams, K. R., Lai, P. L., Wang, X., Montazami, R., and Hashemi, N. N., "Viability of neural cells on 3D printed graphene bioelectronics," Biosensors 9, 112 (2019).

[65] Aykar, S. S., Reynolds, D. E., McNamara, M. C., and Hashemi, N. N., "Manufacturing of poly (ethylene glycol diacrylate)-based hollow microvessels using microfluidics," RSC Adv. 10, 4095-4102 (2020).

[66] AlKhatib, H. S., Taha, M. O., Aiedeh, K. M., Bustanji, Y., and Sweileh, B., "Synthesis and in vitro behavior of iron-crosslinked $\mathrm{N}$-methyl and $\mathrm{N}$-benzyl hydroxamated derivatives of alginic acid as controlled release carriers," Eur. Polym. J. 42, 2464-2474 (2006).

[67] McNamara, M. C., Sharifi, F., Okuzono, J., Montazami, R., and Hashemi, N. N., "Microfluidic manufacturing of alginate fibers with encapsulated astrocyte cells," ACS Appl. Bio Mater. 2, 1603-1613 (2019).

[68] Wu, G., Hu, Y., Zhao, J., Lan, T., Wang, D., Liu, Y., and Chen, W., "Ordered and Active Nanochannel Electrode Design for High-Performance Electrochemical Actuator," Small 12, 4986-4992 (2016). 